\title{
Smoking as an aetiological factor in a pedigree with Leber's hereditary optic neuropathy
}

\author{
Kailenn Tsao, Phil A Aitken, Donald R Johns
}

\begin{abstract}
Backgroundlaims-Leber's hereditary optic neuropathy (LHON) is a mitochondrial DNA mediated disease which causes severe visual deficits. Although expressivity of the disease is $100 \%$, penetrance is variable, and environmental factors may influence risk of becoming symptomatic. The causative relation between cigarette smoking and disease penetrance was examined.

Methods-The incidence of smoking in 65 age matched family members of one LHON pedigree was retrospectively obtained. Smoking in groups which expressed disease was compared with those which did not. Male subgroups were analysed separately in addition to combined sex groups.

Results-The association between smoking and disease penetrance was significant in all subgroups ( $p$ values from $p=0.0009$ to $p=0.0001,95 \%$ confidence intervals). Disease penetrance was higher in males than females. The association was weaker in the male group than combined sex groups ( $p$ values from $p=0.0146$ to $\mathrm{p}=0.0008,95 \%$ confidence intervals), probably because of elimination of female asymptomatic non-smokers in the comparison groups. The association was strengthened in older age groups and in groups which smoked more heavily.

Conclusions-Smoking is significantly associated with disease penetrance in this LHON pedigree. Degree of smoking and number of years smoked correlate with increased risk of developing symptoms. (Br f Ophthalmol 1999;83:577-581)
\end{abstract}

Leber's hereditary optic neuropathy (LHON) is a mitochondrial DNA (mtDNA) mediated disease which usually affects people in their mid 20s. Members of an affected pedigree possess one or more specific mitochondrial DNA point mutations which predispose them to a bilateral optic neuropathy and severe visual loss. ${ }^{12}$ Although expressivity of the disease is $100 \%$, penetrance within Leber's families is variable, and environmental factors may influence the risk of becoming symptomatic. Smoking, drinking, diet, exposure to toxins, and head trauma have been among the epigenetic factors suspected of increasing the penetrance of LHON. ${ }^{13-7}$

No statistical analysis implicating cigarette smoking as an initiating factor in LHON penetrance has been published. However, physicians anecdotally note the increased incidence of smoking in patients who develop symptoms of LHON. Furthermore, cases may be mislabelled as tobacco-alcohol amblyopia when both patient and physician are unaware of the patient's hereditary background. ${ }^{5}$

To learn if there is any correlation between smoking and disease penetrance, we examined the smoking habits in one pedigree proved to carry the LHON trait. Our tested hypothesis was that smoking statistically increases the risk of disease penetrance in individuals who carry the LHON trait.

Subjects and methods

A six generation LHON pedigree of maternally related family members was identified based upon a 25 year old male proband (Fig 1). The proband presented with acute vision loss and a 13 year history of heavy smoking. He was found to have specific class I (mt11778), and class II (mt13708) mitochondrial mutations. ${ }^{6}$ All major arms of the family were found to possess LHON mutations identical to those of the proband. Information about past medical and social history, smoking habits, and other possible disease related questions were retrospectively obtained by telephone interviews and questionnaires (see appendix). We were able to obtain information about 65 family members.

Symptomatic and non-symptomatic groups were age matched. The standard pack year method was used to measure the incidence and degree of smoking. The incidence of smoking was compared with disease penetrance at two designated critical ages. Cigarette consumption by age 25 was analysed because that is the usual age by which symptoms of LHON occur. ${ }^{13}$ Cigarette consumption by age 35 was analysed because that is the average age at which symptoms of LHON occurred in this pedigree. For each designated critical age, all symptomatic LHON individuals were used in the analysis, and only non-symptomatic individuals who were at least as old as the designated critical age were used. Individuals who were not at least as old as the designated critical age would not yet have had equal time and opportunity to develop symptoms. Counting these individuals in the analysis would bias the study towards acceptance of the null hypothesis-that is, there is no correlation between cigarette smoking and disease penetrance.

At each designated critical age, two different smoking classifications were used in the analysis to help establish a dose-response relation. In smoking classification 1 , subjects were designated as smokers if they smoked as much or 
Table 1 Contingency table comparing smoking incidence in symptomatic and non-symptomatic LHON individuals at two designated critical ages using two different smoking classifications

\begin{tabular}{|c|c|c|c|c|c|c|c|}
\hline Group & Designated critical age & Pack year cut off & & Symptomatic & Non-symptomatic & Total & Fisher's exact $p$ value \\
\hline \multirow[t]{3}{*}{$\mathrm{M}, \mathrm{F}$} & 25 & 0.5 pack years (smoking classification 1 ) & Smoke & 10 & 23 & 33 & 0.0009 \\
\hline & & & No smoke & 0 & 32 & 32 & \\
\hline & & & Total & 10 & 55 & 65 & \\
\hline \multirow[t]{3}{*}{$\mathrm{M}, \mathrm{F}$} & 25 & 3.5 pack years (smoking classification 2 ) & Smoke & 10 & 17 & 27 & 0.0001 \\
\hline & & & No smoke & 0 & 38 & 38 & \\
\hline & & & Total & 10 & 55 & 65 & \\
\hline \multirow[t]{3}{*}{ Males } & 25 & 0.5 pack years (smoking classification 1 ) & Smoke & 9 & 14 & 23 & 0.0146 \\
\hline & & & No smoke & 0 & 12 & 12 & \\
\hline & & & Total & 9 & 26 & 35 & \\
\hline \multirow[t]{3}{*}{ Males } & 25 & 3.5 pack years (smoking classification 2 ) & Smoke & 9 & 9 & 18 & 0.001 \\
\hline & & & No smoke & 0 & 17 & 17 & \\
\hline & & & Total & 9 & 26 & 35 & \\
\hline \multirow[t]{3}{*}{$M, F$} & 35 & 3.75 pack years (smoking classification 1 ) & Smoke & 10 & 16 & 26 & 0.0001 \\
\hline & & & No smoke & 0 & 32 & 32 & \\
\hline & & & Total & 10 & 48 & 58 & \\
\hline \multirow[t]{3}{*}{$M, F$} & 35 & 8.5 pack years (smoking classification 2 ) & Smoke & 10 & 12 & 22 & 0.0001 \\
\hline & & & No smoke & 0 & 36 & 36 & \\
\hline & & & Total & 10 & 48 & 58 & \\
\hline \multirow[t]{3}{*}{ Males } & 35 & 3.75 pack years (smoking classification 1 ) & Smoke & 9 & 10 & 19 & 0.0051 \\
\hline & & & No smoke & 0 & 12 & 12 & \\
\hline & & & Total & 9 & 22 & 31 & \\
\hline \multirow[t]{3}{*}{ Males } & 35 & 8.5 pack years (smoking classification 2 ) & Smoke & 9 & 7 & 16 & 0.0008 \\
\hline & & & No smoke & 0 & 15 & 15 & \\
\hline & & & Total & 9 & 22 & 31 & \\
\hline
\end{tabular}

more than the least number of cigarettes smoked by any individual, symptomatic or asymptomatic. In smoking classification 2, subjects were designated as smokers if they smoked as much or more than the least number of cigarettes smoked by any symptomatic LHON individual. If subjects were symptomatic before the designated critical age, pack years calculated included only those years smoked up to the time of disease penetrance. Subjects were automatically classified as nonsmokers if they did not have a history of smoking on a daily basis for at least 1 year.

Since molecular confirmation of LHON had only been widely available within the last few years preceding the study, subjects were counted as symptomatic with LHON if (1) they carried a diagnosis of tobacco-alcohol amblyopia, bilateral idiopathic non-resolving optic neuritis or LHON, and, (2) they had a disease course which was consistent with LHON. The above criteria were confirmed in each case by careful examination of medical records and personal communication with the patient's physician.

Since penetrance of the disease is generally noted to be higher in males, they were analysed separately as well as with the total group. Contingency tables were constructed using symp- tomatic and non-symptomatic individuals as comparison groups. Fisher's exact test was used to analyse the incidence of smoking between comparison groups. The results of the analyses are summarised in Table 1.

\section{Results}

TOTAL SAMPLE GROUP

Smoking was analysed in 65 family members at the 25 year old designated critical age, of whom $10(15 \%)$ were symptomatic. Smoking was analysed in 58 family members at the 35 year old designated critical age, of whom 10 $(17 \%)$ were symptomatic. Minimal pack years smoked by any individual smoker was 0.5 pack years. Minimal pack years smoked by any symptomatic individual was 3.5 pack years. The percentage of asymptomatic family members who had a history of smoking ranged from $25 \%$ to $42 \%$ (calculated according to the two smoking classifications at the two different age groups). The percentage of symptomatic family members who had a history of smoking was $100 \%$ in every subgroup (Fig 2). There was a significant correlation between incidence of smoking and penetrance of LHON, with two sided $p$ values ranging from $p=0.0009$ to $\mathrm{p}=0.0001(95 \%$ confidence intervals $)$. Signifi-

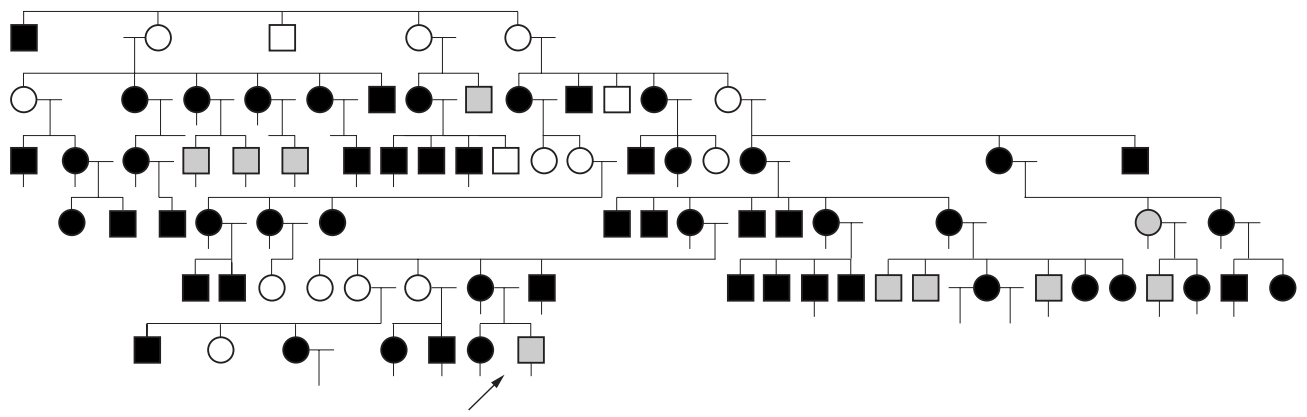

Solid objects, maternally related family members in the study Grey fill objects, symptomatic family members

Objects with tails, blood obtained for mitochondrial analysis Arrow, proband

Figure 1 A six generation pedigree of maternally related family members possessing the LHON trait. 
Figure 2 Comparison of percentage of smokers in symptomatic $(S x)$ and non-symptomatic (Nsx) LHON groups at designated ages (DA) 25 and 35 . In the male subgroups and total groups of each designated critical age, all symptomatic individuals were smokers while only a percentage of the non-symptomatic individuals were smokers.

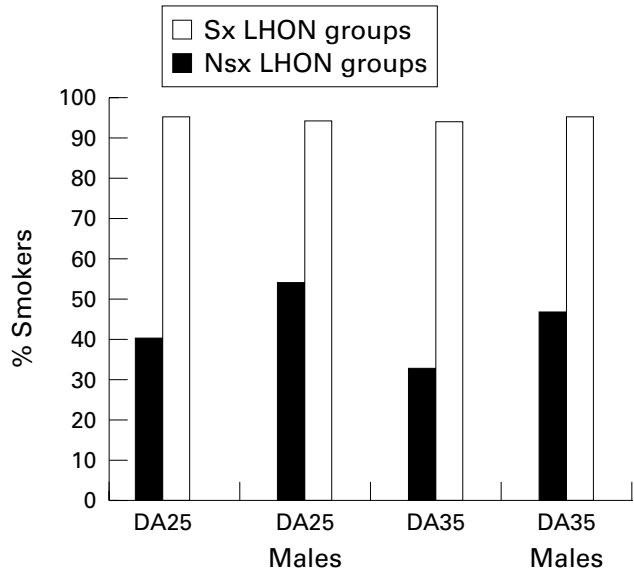

cance of the correlation remained the same or increased at older $v$ younger designated critical ages. Significance of the correlation increased when using smoking classification 2 to designate smokers $v$ non-smokers. Relative risk of smoking was noted to be infinity, since no nonsmokers developed symptoms.

MALE SAMPLE GROUP

Smoking was analysed in 35 male family members at the 25 year old designated critical age, of which nine $(26 \%)$ members were symptomatic. Smoking was analysed in 31 family members at the 35 year old designated critical age, of which nine $(29 \%)$ were symptomatic. Minimal pack years smoked by any individual smoker was 0.5 pack years. Minimal pack years smoked by any symptomatic individual was 3.5 pack years. The percentage of asymptomatic male family members who had a history of smoking ranged from $32 \%$ to $54 \%$ (calculated according to the two smoking classifications at the two different age groups). The percentage of symptomatic male family members who had a history of smoking was $100 \%$ in every subgroup (Fig 3). There was significant correlation between incidence of smoking and penetrance of LHON, with two sided $p$ values ranging from $\mathrm{p}=0.0146$ to $\mathrm{p}=0.0008 \quad(95 \%$ confidence intervals). Significance of the correlation increased at older $v$ younger designated critical ages. Significance of the correlation increased when using smoking classification 2 to designate smokers $v$ non-smokers. In each corresponding subgroup the correlation was weaker in the male group $v$ the total group, probably because of elimination of female asymptomatic non-smokers in the comparison groups. There were far fewer symptomatic females than there were males $(3 \% v 26 \%)$. However, females smoked less overall, and those who did smoke tended to smoke less than their male counterparts. Relative risk of smoking was infinity, since no non-smokers developed symptoms of the disease.

\section{Discussion}

In this LHON pedigree, smoking is correlated significantly with disease penetrance in the total population sampled as well as in the male subgroup. In this small study using Fisher's exact test, degree of significance can be used to

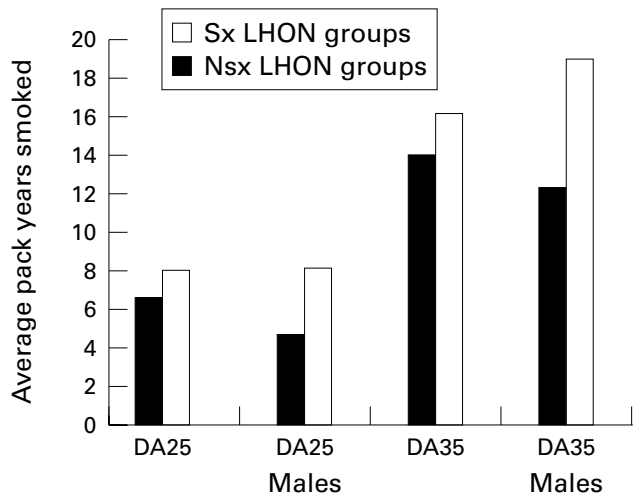

Figure 3 Comparison of average pack years smoked by symptomatic $(S x)$ and non-symptomatic (Nsx) LHON groups at designated ages (DA) 25 and 35. Symptomatic groups at designated ages (DA) 25 and 35 . Symptomatic
individuals smoked more pack years than non-symptomatic smoking individuals in the male subgroups as well as in the total groups at each designated critical age.

comment on the association between the variables. $^{89}$ By using two different smoking classifications, it was shown that proportionally more symptomatic LHON individuals smoked (Fig 2), and smoked more (Fig 3), than non-symptomatic individuals by the designated critical ages. In fact, no non-smokers developed symptoms of LHON. This implies that there may be a threshold of smoking below which symptoms of LHON do not occur in this pedigree. The correlation was strengthened at older $v$ younger designated critical ages in seven of eight comparison groups, probably because younger asymptomatic smokers were not included in the analysis in the older age groups. This implies a high likelihood that significant numbers of asymptomatic young smokers will become symptomatic as they approach the older designated critical ages. There was significantly decreased disease penetrance in females $v$ males, which paralleled a decreased incidence of smoking in females. Females who did smoke tended to start smoking later in life and smoked fewer pack years. However, endogenous factors may also contribute to the sex asymmetry which previously had been attributed to an $\mathrm{X}$ linked genetic factor. ${ }^{10}$ The correlation was weakened in the male group $v$ the total group probably because of elimination of female asymptomatic nonsmokers in the male comparison groups.

Sources of bias in the study may have been introduced at the level of subjective recall of information. Although subjects were carefully interviewed to ascertain accurate data, there may have been a tendency for subjects to exaggerate smoking history if there was a personal history of visual impairment. ${ }^{11}$ Prospective studies examining pedigrees over the span of generations would eliminate such bias. In addition, the pack year method used to quantify smoking habits did not take into account variations in smoking habits over the years. It may be that a crucial amount of cigarette smoking at a critical age is necessary to trigger the symptoms of LHON. The possibility that childhood exposure to second hand cigarette smoke may play a role in expression of disease could not be ruled out. Information about brand of cigarettes, nicotine, tar content, 
inhalation habits, alcohol consumption, and others was insufficient for analysis. ${ }^{10} 12$

In recent years, significant inroads have been made towards identifying and characterising LHON on a genetic basis. However, little progress has been made in terms of its treatment or prevention. Identifying environ- mental factors which may significantly influence penetrance of the disease is of importance to those at risk. Results of this study indicate that smoking may be an important risk factor in some pedigrees. Further research into the detection, prevention, and treatment of this genetic disease is warranted.

\section{Appendix}

Questionnaire used to obtain information about past medical and social history, smoking habits, and other possible disease related questions.

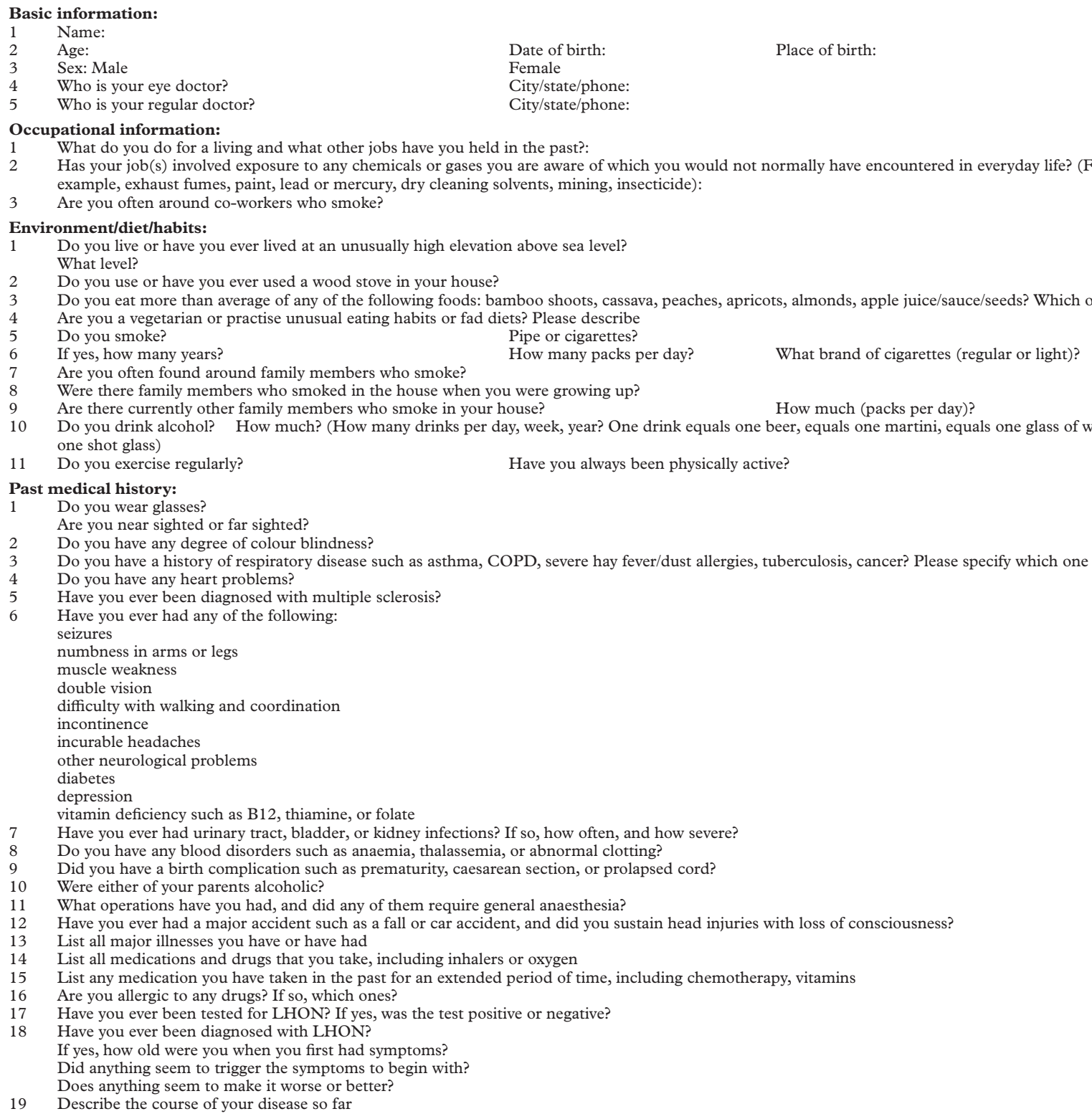

Family history:

Are you married?

How many children do you have?

3 Do your children have any significant visual problems or wear glasses? Please describe

4 Please list as many family members as you can, their relation to you, and whether they have any significant visual problems that you are aware of. Name: Relation: Vision:

5 Is there anything else you feel is important to let us know that we have not asked you? (Please use more paper if necessary) 
Statistical consultation and assistance provided by Diantha $\mathrm{B}$ Howard, MS, University of Vermont College of Medicine, Department of Biostatistics.

This work was supported by a grant from the National Eye Institute (ROI EY10864) to DRJ.

1 Johns DR. Seminars in medicine of the Beth Israel Hospital, Boston. Mitochondrial DNA and disease. $N$ Engl $\mathcal{F} \mathrm{Med}$ 1995;333:638-44

2 Kosmorsky G, Johns DR. Neuro-ophthalmic manifestations of mitochondrial DNA disorders: chronic external ophthalmoplegia, Kearns-Sayre syndrome, and Leber's hereditary optic neuropathy. Neurol Clin 1991;9:147-61.

3 Volpe NJ. Lessell S. Leber's hereditary optic neuropathy. Int Ophthalmol Clin 1993;33:153-68.

4 Miller NR. The hereditary optic neuropathies. In: Walsh and Hoyt's Clinical neuro-ophthalmology, 4th Ed. Baltimore: Hoyt's Clincal neuro-ophthalmology,
Williams and Wilkins, 1982:311-20.
5 Cullom ME, Heher KL, Miller NR, et al. Leber's hereditary optic neuropathy masquerading as tobacco-alcohol amoptic neuropathy masquerading as tobacco-a
blyopia. Arch Ophthalmol 1993;111:1482-5.

6 Shoffner JM, Wallace DC. Oxidative phosphorylation diseases, disorders of two genomes. Adv Hum Genet 1990;19: eases, dis

7 Wilson J. Cyanide in human disease, a review of clinical and laboratory evidence. Fundam Appl Toxicol 1983;3:397-9.

8 O'Brien PC, Shampo MA. Statistics for clinicians. Mayo Clin Proc 1981;56:18-22.

9 Statistics: GraphPad Instat, Version 2.0, San Diego: 1993. $10 \begin{gathered}\text { Brown MD, Voljavec AJ, Lott MT, et al. Leber's hereditary } \\ \text { optic neuropathy: a model for mitochondrial neurodegen- }\end{gathered}$ erative diseases. FASEB f 1992;6:2791-9.

11 Perez-Stable EJ, Marin G, Marin BV, et al. Misclassification of smoking status by self-reported cigarette consumption. Am Rev Respir Dis 1992;145:53-7.

12 Burns DM. Cigarettes and cigarette smoking. Clin Chest Med 1991;12:631-42. 